Case report

\title{
Panoramic radiography as an auxiliary in detecting patients at risk for cerebrovascular accident (CVA): a case report
}

\author{
Flávio Ricardo Manzi ${ }^{\S}$, Frab Norberto Bóscolo ${ }^{\dagger}$, Solange Maria de Almeida ${ }^{\dagger}$ \\ and Francisco Haiter Neto ${ }^{\dagger}$ \\ ${ }^{\S}$ Department of Oral Radiology, School of Dentistry of Belo Horizonte, PUC-Minas, MG, Brazil \\ ${ }^{\dagger}$ Department of Oral Radiology, School of Dentistry of Piracicaba, University of Campinas, SP, Brazil
}

(Received 22 November 2002 and accepted 20 June 2003)

\begin{abstract}
Most cerebrovascular accidents (CVAs), commonly referred to as noncardiogenic strokes, occur as a result of atherosclerosis involving the common, internal and external carotids arteries, due to atheroma formation. Several factors influence atheroma formation, such as hypertension, smoking, diabetes mellitus, hypercholesterolemia, obesity and sedentary lifestyle among others. When atheromas are positioned inside the vessel lumen, they alter the flow of blood, causing the stroke. These atheromas, that are calcified plaques, can be observed in panoramic radiography. (J. Oral Sci. 45, 177-180, 2003)
\end{abstract}

Key words: panoramic radiography; cerebrovascular accident; stroke, atheroma.

\section{Case Report}

A 68-year-old man came to the Radiology Clinic of the Piracicaba Dentistry School at the State University of Campinas for panoramic radiography screening prior to prosthetic treatment. Panoramic radiography showed several bilateral nodular radiopaque masses located inferior and posterior to the angle of the jaw, above the hyoid bone (Fig. 1). As the vertebral bodies were not visible, a further anteroposterior projection with the patient's mouth shut was taken, for the purpose of differential diagnosis. This radiograph verified that these masses were located

Correspondence to Dr. Flávio Ricardo Manzi, Av Paes de Barros, 1340, apt 62, Mooca, CEP: 03114-000, São Paulo, SP, Brazil E-mail: flaviomanzi@hotmail.com in the intervertebral space between $\mathrm{C} 3$ and $\mathrm{C} 4$ (Fig. 2).

Physical examination revealed no neck bruits and the patient reported no history of overt transient ischemic

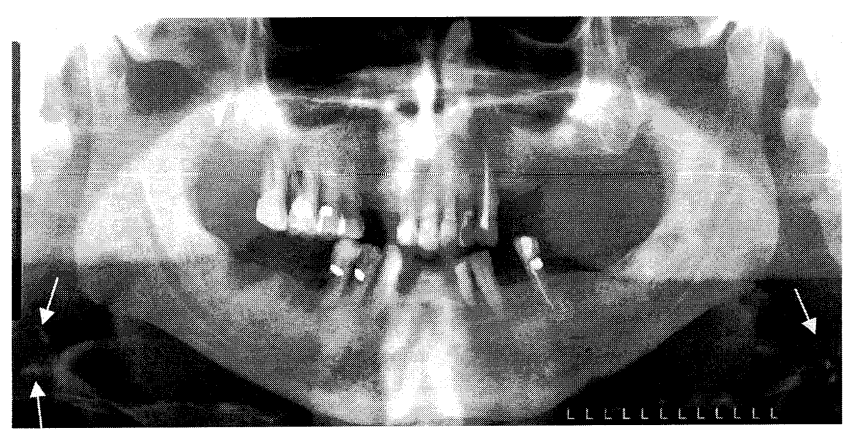

Fig. 1 Standard panoramic dental radiograph of 68-year-old man with calcified carotid arterial plaque visible bilaterally in soft tissues of the neck, inferior to the angle of the mandible, adjacent to the hyoid bone (arrow).

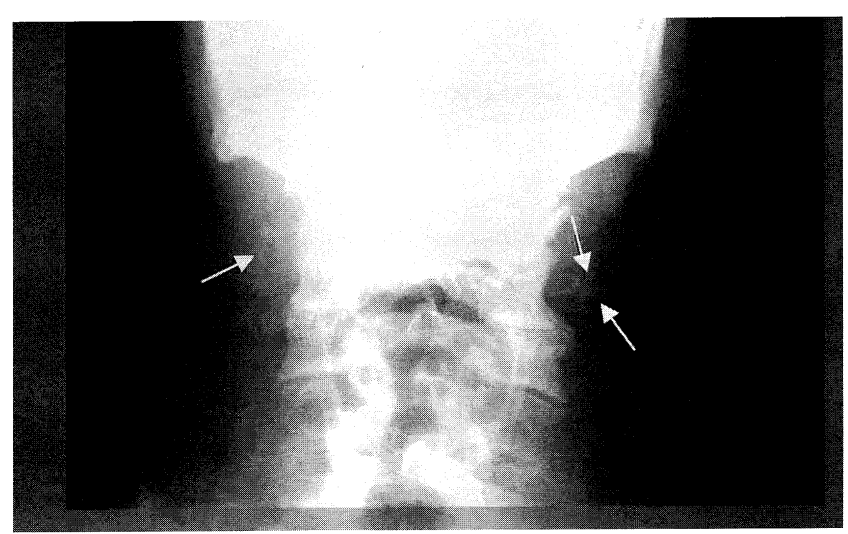

Fig. 2 Standard anteroposterior projection with calcified carotid arterial plaque visible bilaterally, located in the intervertebral space between C3 and C4 (arrow). 
attacks. Risk factors for cerebrovascular disease included a history of 42 years of smoking, hypertension controlled through drugs, and diet-controlled diabetes. He had no history of stroke, transient ischemic attacks or hospitalisation.

After the calcified atheromas were detected, the patient underwent a color flow Doppler scan of the carotid artery (Fig. 3). It was confirmed that the radiopaque masses seen in the panoramic radiographs were atheromas located at the right bifurcation (bulb) of the common and internal carotid artery and the left bulb of the common carotid artery. The Doppler study also determined that these lesions had decreased the luminal vessel diameter by approximately $30 \%$ (right side) and $40 \%$ (left side).

As the obstruction was less than $60 \%$, the patient was placed on aspirin therapy and diet to reduce obesity, while continuing to control blood glucose levels and arterial pressure.

\section{Discussion}

Cerebrovascular accident (CVA) is one of the most common causes of death in the world and can be divided in two types: haemorrhagic and thromboembolic. About $15 \%$ of CVAs are haemorrhagic, occurring as a result of ruptured vessels. This mechanism of causation shows a predilection for intracranial vessels (1,2). Approximately $85 \%$ of strokes occur when a thrombus or clot occludes the vessel lumen and results in ischemia distal to the site of the occlusion. In most cases (60\%), thromboembolic strokes originate from plaques formed in the bifurcation of the carotid. The middle cerebral artery, a direct continuation of the internal carotid artery, is the vessel most

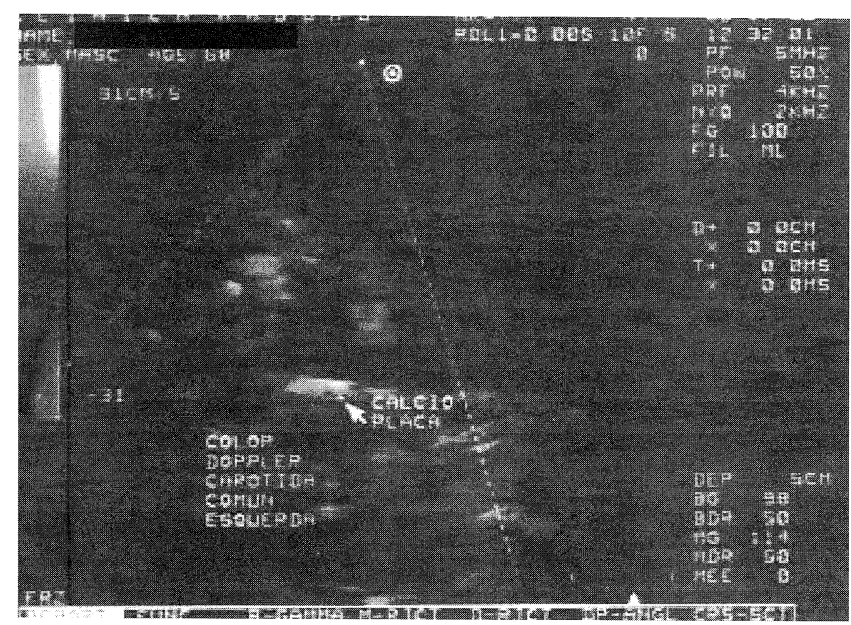

Fig. 3 Duplex Doppler ultrasound shows that plaque has decreased the luminal vessel diameter by around $30 \%$ in the right bulb of the common and internal carotid artery. susceptible to being occluded by a clot in the carotid bifurcation (1).

The formation of atherosclerotic plaques begins through injuries to the endothelium cells, caused by hypertension and hypercholesterolemia resulting from smoking, diabetes mellitus, age and other factors. Serum lipoproteins permeate the damaged endothelium and lodge in the vessel intima, while platelet components stimulate the growth factor for the proliferation of smooth muscle cells. When these atherosclerotic plaques became encrusted with calcium salts, they are known as atheromas. Plaques undergo repeated cycles of deterioration and repair, including intraplaque hemorrhage followed by ulceration through the endothelium. When this happens, collagen fibers are exposed, which leads to the development of a mural thrombus. In some patients, embolization of the thrombus obstructs the intracranial artery and leads to thromboembolic CVA. In other patients, cerebral ischemia occurs when the diameter of the atheroma increases, thus reducing the vessel lumen. This decreases the blood flow distally in the artery (1-5).

There are many risk factors for the development of atheromas, including increasing age (above 50 years) (1), obesity $(1,3,4)$, hypertension (1,3-5), prior transient ischemic attacks $(4,6)$, prior stroke $(3,4,6)$, alcohol abuse $(1,5,7)$, hypercholesterolemia (4), elevated serum triglicerides (4), smoking (1,3-5,8,9), diabetes mellitus $(1,4,10,11)$, and sedentary lifestyle $(1,4)$.

When atherosclerotic lesions are partially calcified, they can be observed in panoramic radiography $(1,2,4-6,11$ 13). The image of the atheroma may appear either as a nodular radiopaque mass (Fig. 4) or as two radiopaque vertical lines inferiorly $(1.5-4.0 \mathrm{~cm}$ inferior to the angle of the mandible) and posteriorly to the $\mathrm{C} 3$ and $\mathrm{C} 4$ vertebral bodies, separate and distinct from other radiopaque

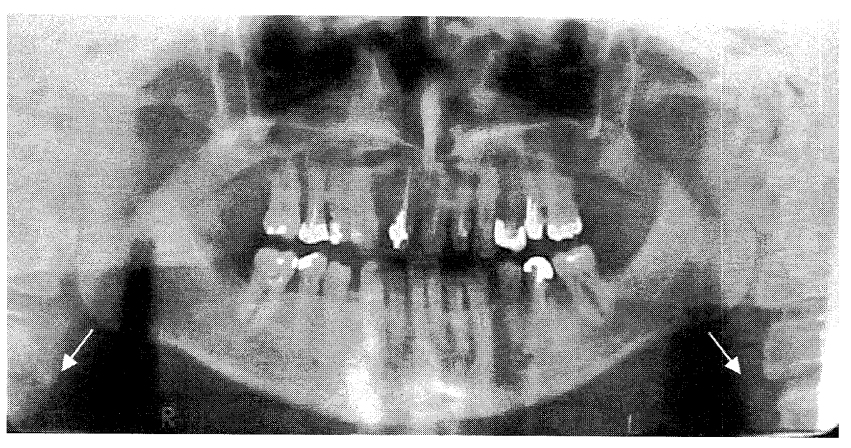

Fig. 4 Standard panoramic dental radiograph of a 60-year-old woman with calcified carotid arterial plaque visible bilaterally in soft tissues of the neck, inferior to the angle of the mandible, adjacent to the hyoid bone and C3C4 (arrow). 
structures (Fig. 5). However, panoramic radiography is limited to the identification of the atheroma, and cannot evaluate its exact location and degree of occlusion. Contrast angiography is also used for the confirmation of the presence of calcifications, its nature and extension $(1,12)$. However, the high cost, the potential for serious complications, and the limited information obtained by this method has stimulated the development of other techniques which are not invasive, such as color flow Doppler studies (ultra-sound) and Doppler spectral analysis (Spectral Doppler Scanner) $(1,5,12)$. These techniques are relatively inexpensive and have a low risk of complications and low mortality rates, while supplying sophisticated images. Computed tomography $(12,14)$ and thermography $(15)$ can also be used to study carotid calcifications.

In this case, an anteroposterior projection with the patient's mouth shut was taken to confirm that the radiopaque nodular masses were located in the intervertebral space between $\mathrm{C} 3$ and $\mathrm{C} 4$. This projection can be used when the vertebral bodies are not detectable in the panoramic radiograph.

Differential diagnosis of the image of the atheroma includes the anatomical structures of the neck area, such as the hyoid bone, soft tissue of the tongue and auricle, the epiglottis, and lesions such as rhinoliths, antraliths, calcified stylomandibular ligaments, calcified stylohyoid ligaments, sialoliths, tonsilloliths, phleboliths and calcified lymphatic nodules $(4,16)$.

If the atherosclerotic lesions occlude more than $60 \%$ of the diameter of the carotid artery, a carotid endoarterectomy is necessary to remove the plaques. Atherosclerotic lesions with a smaller degree of blockage should be treated with aspirin or triclopidine to decrease platelet aggregation and associated mural thrombus formation. Medications to control blood pressure, glucose and cholesterol levels

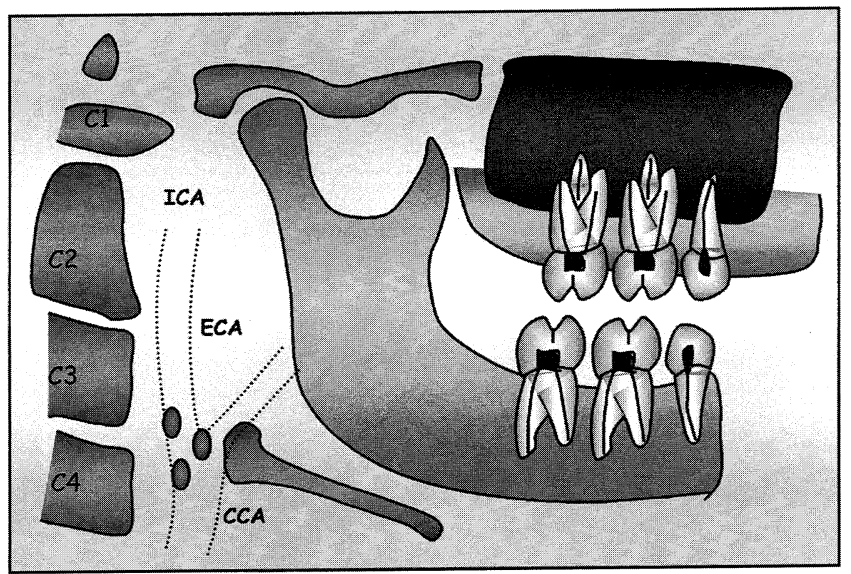

Fig. 5 Schematic representation of panoramic radiograph indicates location of the atheroma. should also be prescribed when indicated. Changes in lifestyle are recommended, with the aim of eliminating unhealthy habits $(2,4,5)$.

Panoramic radiography presents a satisfactory image for the identification of asymptomatic patients with risk of developing CVA. Dentists should be trained to identify these calcifications in the carotid artery through panoramic radiography. If there are doubts about the diagnosis, a radiologist should be consulted to obtain a second opinion. After a diagnosis is agreed upon, the patient should be referred to a physician for evaluation of the risk of developing a CVA and to receive the correct treatment. Treatment of the condition before a stroke can occur is of vital importance because it is known that the damage caused by a CVA is irreparable in most cases. This will result in a decrease in the morbidity and mortality of CVA $(4,5)$.

\section{References}

1. Carter LC, Haller AD, Nadarajah V, Calamel AD, Aguirre A (1997) Use of panoramic radiography among an ambulatory dental population to detect patient at risk of stroke. J Am Dent Assoc 128, 977-984

2. Friedlander AH, Manesh F, Wasterlain CG (1994) Prevalence of detectable carotid artery calcification on panoramic radiographs of recent stroke victims. Oral Surg Oral Med Oral Pathol 77, 669-673

3. Doris I, Dobranowski J, Franchetto A, Jaeschke R (1993) The relevance of detecting carotid artery calcification on plain radiograph. Stroke $24,1330-$ 1334

4. Friedlander AH (1995) Panoramic radiography: the differential diagnosis of carotid artery atheromas. Spec Care Dent 15, 223-227

5. Friedlander AH, Friedlander IK (1996) Panoramic dental radiography: an aid in detecting individuals prone to stroke. Br Dent J 181, 23-26

6. Barret-Connor E (1990) Obesity, hypertension and stroke. Clin Exp Hypertens A 12, 769-782

7. Friedlander AH, Lander A (1981) Panoramic radiographic identification of carotid arterial plaques. Oral Surg Oral Med Oral Pathol 52, 102-104

8. Scwartz CJ, Valente AJ, Sprague EA, Kelley JL, Cayatte AJ, Rozek MM (1992) Pathogenesis of the atherosclerotic lesion: Implications for diabetes mellitus. Diabetes Care 15, 1156-1167

9. Whisnant JP, Homer D, Ingall TJ, Baker HL Jr, O'Fallon WM, Weibers DO (1990) Duration of cigarette smoking is the strongest predictor of several extracranial carotid artery atherosclerosis. Stroke 21, 
707-714

10. Kawamori R, Yamasaki Y, Matsushima H, Nishizawa H, Nao K, Hougaku H, Maeda H, Handa N, Matamoto M, Kamada T (1992) Prevalence of carotid atherosclerosis in diabetic patients. Ultrasound high-resolution B-mode imaging on carotid arteries. Diabetes Care 15, 1290-1294

11. Lewis D, Brooks S (1999) Carotid artery calcification in a general dental population: a retrospective study of panoramic radiographs. Gen Dent 47, 98-103

12. Friedlander AH, Gratt BM (1994) Panoramic dental radiography as an aid in detecting patients at risk for stroke. J Oral Maxillofac Surg 52, 1257-1262

13. Carter LC, Tsimidus K, Fabiano J (1998) Carotid calcifications on panoramic radiography identify an asymptomatic male patient at risk for stroke. A case report. Oral Surg Oral Med Oral Pathol Oral Radiol Endod 85, 119-122

14. Culebras A, Otero C, Toledo JR, Rubin BS (1989) Computed tomographic study of cervical carotid calcification. Stroke 20, 1472-1476

15. Gratt BM, Sickles EA (1991) Future applications of electronic thermography. J Am Dent Assoc 122, 28-36

16. Friedlander AH, Baker JD (1994) Panoramic dental radiography: an aid in detecting patients at risk of cerebrovascular accident. J Am Dent Assoc 125 1598-1603 